\title{
Accidents involving pedestrians with their backs to traffic or facing traffic: an evaluation of crash characteristics and injuries
}

\author{
Ping-Ling Chen ${ }^{1}$, Rong-Chang Jou ${ }^{2}$, Wafaa Saleh ${ }^{3}$ and Chih-Wei Pai ${ }^{1} *$ \\ ${ }^{1}$ Institute of Injury Prevention and Control, College of Public Health and Nutrition, Taipei Medical University, Taipei, \\ Taiwan \\ ${ }^{2}$ Department of Civil Engineering, College of Science and Technology, National Chi Nan University, Nantou City, \\ Taiwan \\ ${ }^{3}$ Transport Research Institute, Edinburgh Napier University, Edinburgh, U.K.
}

\begin{abstract}
SUMMARY
This paper examines pedestrian anatomical injuries and crash characteristics in back-to-traffic and facingtraffic crashes. Pedestrian crashes involving pedestrians walking along streets (i.e. with their backs to traffic or facing traffic) have been overlooked in literature. Although this is not the most frequent type of crash, the crash consequence to pedestrians is a safety concern. Combining Taiwan A1A2 police-reported accident data and data from the National Health Insurance Database from years 2003-2013, this paper examines anatomical injuries and crash characteristics in back-to-traffic and facing-traffic crashes. There were a total of 830 and 2267 pedestrian casualties in back-to-traffic and facing-traffic crashes respectively. The injuries sustained by pedestrians and crash characteristics of these two crash types were compared with those of other crossing types of crashes (nearside crash, nearside dart-out crash, offside crash, and offside dart-out crash). Odds of various injuries to body regions were estimated using logistic regressions. Key findings include that the percentage of fatalities in back-to-traffic crashes is the highest; logistic models reveal that pedestrians in back-to-traffic crashes sustained more head, neck, and spinal injuries than did pedestrians in other crash types, and unlit darkness and non-built-up roadways were associated with an increased risk of pedestrian head injuries. Several crash features (e.g. unlit darkness, overtaking manoeuvres, phone use by pedestrians and drivers, and intoxicated drivers) are more frequently evident in back-to-traffic crashes than in other types of crashes. The current research suggests that in terms of crash consequence, facing traffic is safer than back to traffic. Copyright $@ 2016$ John Wiley \& Sons, Ltd.
\end{abstract}

KEY WORDS: pedestrian accident; crash characteristic; injury; facing-traffic; back-to-traffic

\section{INTRODUCTION}

Pedestrians on roadways are the most vulnerable to injuries as a result of crashes with moving vehicles; thus, accidents involving pedestrians and vehicles represent a significant problem. In Taiwan, for example, approximately 13000 accidents involving pedestrians and vehicles occurred in 2013, and 415 pedestrians were killed. Pedestrian deaths account for approximately $13 \%$ of all traffic fatalities each year [1].

Extensive research has well documented that most pedestrian accidents take place on urban roadways [2], primarily because of frequent walking and higher traffic volume. However, injuries sustained by pedestrians in accidents that occur on rural roadways tend to be more severe than those that occur on urban roadways, possibly as a result of a higher collision velocity [3], drivers' not expecting to encounter pedestrians [4], and lower efficiency of emergency services travelling over long distances [5].

*Correspondence to: Chih-Wei Pai, Institute of Injury Prevention and Control, College of Public Health and Nutrition, Taipei Medical University, Taipei, Taiwan. E-mail: Zohal.Hessami@uni-konstanz.de 
Research into accidents where pedestrians attempt to cross streets at midblock locations and intersections has suggested that crossing the road is the most frequent event in fatal pedestrian accidents [2]. In addition, midblock crossing accidents can be more severe than those at intersections. Studies of pedestrian fatality risk in accidents at unsignallised zebra crosswalks [6] reported that factors such as male pedestrians, unlit darkness, non built-up area, mid-block crosswalk location, and summer time period increased the probability of pedestrian death.

Elvik et al. [7] investigated the factors influencing safety in a sample of marked pedestrian crossings in the city of Oslo. They reported several findings: an increase in the number of pedestrians is associated with a lower risk of accident for each pedestrian; crossings located in four-leg junctions or roundabouts had more accidents than crossings located in three-leg junctions or on sections between junctions; a tendency was seen for risk to be higher when crossing the road outside a marked crossing than when crossing the road at the crossing, and increased speed was associated with an increased number of accidents.

Three of the most common crash configurations involving pedestrians and vehicles are offside (left side, the side of vehicle nearest the centreline), nearside (right side, the side of vehicle nearest the kerb), and dart-out accidents. Researchers have suggested that older pedestrians are over-involved in offside crashes [8]. This is likely because older people walk more slowly than younger people do and thus have more difficulty crossing roads before the arrival of traffic or before traffic signals change. Moreover, because of older people's diminished attention capacity, they have more difficulty judging two streams (or above) of traffic before crossing roads than younger people do. Older children and younger children are found to be over-involved in nearside accidents and dart-out accidents, respectively, where children enter roadways without considering traffic sensibly [9].

Light conditions also create crucial problems for pedestrian safety; at nights, drivers are often unable to recognise and respond to pedestrians from a safe distance [10]. Past studies have reported that the average fatal injury risk is several times higher in dark conditions than in daylight conditions [11]. A considerable amount of research has suggested that the increased incidence of crashes involving pedestrians at nights primarily results from lower illumination rather than from other factors that vary between day and night such as driver fatigue and alcohol use [12].

There exist fewer studies of pedestrian accidents, in which pedestrians were walking along the streets. Among the few studies, alcohol-impaired pedestrians were found to be over-presented in walking-along crashes [2]. Out of 100 fatally injured pedestrians who were walking on the carriageway, 75 were not facing the traffic. Pedestrians are therefore killed less often when they are facing the traffic. Fontaine and Gourlet [2] indicated that without exposure data, however, it is difficult to asses any over-risk related to the direction in which the pedestrian was walking. More recently, Luoma and Peltola [4] have investigated 18 fatal pedestrian accidents and 87 nonfatal accidents in Finland, suggesting that facing traffic compared with walking with traffic resulted in a $77 \%$ decrease in the number of fatal and nonfatal pedestrian accidents. According to Luoma and Peltola [4], likely reasons for this conclusion include that facing traffic is safer for pedestrians because of the visual information it provides about vehicles in the lane closest to them. They further indicated that the benefit effect of facing traffic for pedestrians was greater on main roads than on secondary roads.

Regarding body injured regions by pedestrians, comparatively consistent findings have been reported in past studies [13-17] — research has suggested that head/lower/upper extremities injuries are the most common injury regions. Head injuries tend to be more life-threatening, while lower/upper extremities are likely to lead to long-term disability [18].

Martin et al. [14] reported that pelvic injuries were much more common for women. The most severe injuries (AIS4+) were mostly to the head and thorax, for all groups of road users. More specifically, the risk of sustaining an AIS2+ thoracic injury was higher in a collision with a multipurpose vehicle. Maki et al. [19] concluded that pedestrians and bicyclists suffered fewer fatalities in collisions with sedans than in collisions with minivans or SUVs, which have high bonnet leading edges. Zhao et al. [20] pointed out that a higher risk of head injury was associated with being female, age over 60 , impact speeds over $40 \mathrm{~km} / \mathrm{h}$, and a likelihood of the victim's head striking the vehicle rather than the ground. Impact speeds of over $40 \mathrm{~km} / \mathrm{h}$ and head contact site on windscreen frame/A pillar retained a strong association with severe head injury (AIS 5-6) rate. Mizuno and Kajzer [21] reported that in collisions with mini vans, the injury risk to the head is higher, as a result of a head 
impact against stiff structures such as windshield frames. When pedestrians are struck by a car with a short hood length, their heads are likely to strike into or around the windshield.

Among the multivariate modelling techniques, the logistic regression has been commonly used when the outcome variable is in a binary form (such as fatal versus non-fatal, injury versus non-injury, and one certain injury region). For studies analysing accident/injury severities in bicyclist or pedestrian accidents, the logistic regression model has been frequently estimated when the variable of interest is recorded in binary form (refer to, for example, [22-26]). Generally, these researchers were in an attempt to model the probability of fatalities/severe injuries using a variety of variables such as junction control measures, pre-crash movement of the car, age/gender of bicyclist/pedestrian, and vehicle type. Studies focusing on other road users such as motorcyclists and automobile drivers were also employing the logistic models with considerable success. For instance, Gabella et al. [27], Peek-Asa and Kraus [28], and Zambon and Hasselberg [29] to model the probability of fatalities/severe injuries/severe head injuries using a wide-range of factors such as rider age/gender, helmet use, weather condition, and engine size.

In many countries, including Taiwan, pedestrians are advised to walk facing traffic because this has been suggested to increase pedestrian safety. Official accident statistics in Taiwan show that this crash type, walking along the street, accounts for approximately $10 \%$ of all nonintersection pedestrian accidents, and accidents involving pedestrians walking along streets appear to result in more serious accident consequence to pedestrians than those involving pedestrian midblock crossing do. Although crashes involving pedestrian walking along streets do not constitute the most frequent crash type, injury consequence to pedestrians in appears to be a safety concern. Official accident statistics show that the fatality rates for pedestrians in back-to-traffic crashes and facing-traffic crashes are $6.0 \%$ and $3.4 \%$, respectively, which are higher than those in other crash types [1].

The current research uses a large sample of injured pedestrians for whom crash and injury information is available from police-reported crash data and the National Health Insurance Database and evaluates crash characteristics and injuries sustained by pedestrians in facing-traffic and back-totraffic accidents. Driver and pedestrian features such as alcohol use, phone use, age, and gender as well as crash features, such as temporal, vehicle, and roadway factors were compared for facingtraffic and back-to-traffic accidents as well as for other crossing types of crashes. Body region injuries resulting from facing-traffic and back-to-traffic accidents, and other crash types are described and compared.

The remainder of the current paper is organised as follows. The next section describes the dataset as well as the method used in the current research to clarify crash characteristics and injuries sustained by pedestrians in facing-traffic and back-to-traffic accidents. The research findings, discussion, and implications of the findings are then provided.

\section{METHOD}

\subsection{Data source}

The sources of data in the present study were police-reported crash data and the National Health Insurance Database. The police-reported data (Taiwan A1A2A3 accident data, A1A2) are recorded by the National Police Agency, Taiwan. After every road traffic accident of which police are aware, qualified and experienced police accident investigators complete A1A2 report forms, which comprise three files: accident, vehicle and victim, and contributory factor files.

Accident files contain general information on the times and dates of accidents; weather, road, and light conditions; and road type. Furthermore, vehicle and victim files are used to record information on vehicles, drivers, and victims, such as the age, gender, and injury severity of drivers and victims; vehicle type; first vehicle impact of point; and vehicle manoeuvres. Finally, contributory factor files are used to report likely reasons for accidents. Injury consequence is classified into three levels: A1, A2, and A3. A1 injuries include those sustained by victims who die within 24 hours as a result of an accident, A2 injuries include those sustained by victims who suffer mild or severe injuries but do not die within 24 hours of an accident, and A3 injuries include those sustained by victims who incur only damage to their property damage as a result of an accident. A3 data, however, were not 
considered in the present study because they are deposited by local police agencies, and a vast majority of these data are not acquired by the National Police Agency, Taiwan.

Data on accidents involving pedestrians and vehicles (in this paper, the term 'vehicle' is used to represent any moving motorised vehicle that strikes a pedestrian, including motorcycles, passenger cars, buses and coaches, and heavy goods vehicles (HGVs) from the period 2003-2013) were extracted from the A1A2 dataset. For obtaining additional information such as alcohol use and injured anatomical body regions, driver, and pedestrian IDs were used as identifiers to link the data from the A1A2 dataset with those from the National Health Insurance Database. Only accidents that resulted in pedestrian injuries were considered in this study. A total of 31498 pedestrian casualties were obtained from the two datasets.

Additionally, some data are unlikely to be reported for all crashes, particularly less severe crashes. An example is data on mobile phone usage, which are difficult to obtain. The involved pedestrians or drivers may not honestly tell the truth on phone use. Another example of the data that are unavailable is the presence of sidewalk where the pedestrian crash was recorded. We attempted to trace up the sidewalk data through other sources but failed to do so. Actually, even if the sidewalks are available, pedestrians are occasionally forced to walk on the shoulders of roadways because of vendors and illegal parking of motorcycles/bicycles, etc. (which are common in developing countries).

This study included only accidents that resulted in injuries to pedestrians (i.e. A1A2 accidents). Crashes that did not cause injuries to pedestrians (i.e. A3 accidents) were not included. We believe that the exclusion of A3 accidents is unlikely to influence the results of this study because most crashes involving pedestrians and motorised vehicles cause injuries to pedestrians.

\subsection{Definitions}

This section provides detailed definitions of key variables (e.g. road environment, driver/pedestrian behaviours, and crash types) examined in the analysis. The variable 'speed limit' comprises two categories: built-up roadway (BU: speed limit $\leq 50 \mathrm{~km}$ ) and non-built-up roadway (NBU: speed limit $\geq 60 \mathrm{~km}$ ). The variable 'street light condition' includes several categories: daylight, street light lit/unlit in darkness, and street light unknown. The data for vertical or horizontal curvature record whether vertical or horizontal curvature were present in the scene of crash.

Crashes relating to hit and runs were defined for accidents, in which the drivers fail to stop at the scene of an accident. Data regarding phone use were recorded by the police who interviewed the causalities and other witness. Vehicle's overtaking manoeuvre is a variable of interest, which is defined for a crash in which a driver was executing an overtaking manoeuvre and striking a pedestrian.

The current study compares crash features and injuries sustained by pedestrians in facing-traffic and back-to-traffic accidents with those of other crossing types of crashes (i.e. midblock crossing crashes). Accidents that occurred at intersections were excluded from thus study to avoid the complexity of considering vehicle manoeuvres and other confounding factors. Midblock crossing crashes were classified into four crash types: nearside, nearside dart-out, offside crashes, and offside dart-out crashes. Accidents involving pedestrians walking along streets were classified into two crash types: facing-traffic and back-to-traffic crashes. The six crash types are described in details as follows and illustrated in Figure 1.

A nearside crash (Figure 1a; Crash A) is defined as a collision that occurs when a crossing pedestrian from nearside is struck by an approaching vehicle. A nearside dart-out crash (Figure 1b; Crash B) occurs when a crossing pedestrian from nearside that is obscured or blocked by a parked or stationary vehicle/object. An offside crash (Figure 1c; Crash C) is defined as a collision that occurs when a crossing pedestrian from offside is struck by an approaching vehicle. Furthermore, an offside dart-out crash (Figure 1d; Crash D) occurs when a crossing pedestrian from offside that is obscured or blocked by a parked car or stationary vehicle. A facing-traffic accident (Figure 1e; Crash E) is defined as a crash that occurs when a pedestrian that is walking against is struck by an oncoming vehicle. Finally, a back-to-traffic crash (Figure 1f; Crash F) is defined as a crash that occurs when a pedestrian that is walking with the traffic is struck from behind by a vehicle. The frequency of these six crash types, as well as the crash and pedestrian features, is reported in Table I. Only accidents resulting in injuries to pedestrians were included in the analysis. 
(a)

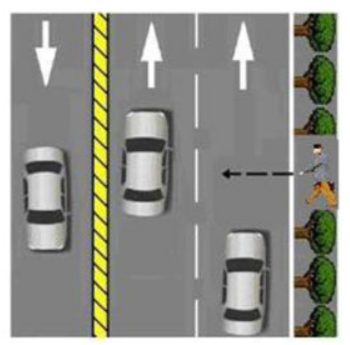

(d)

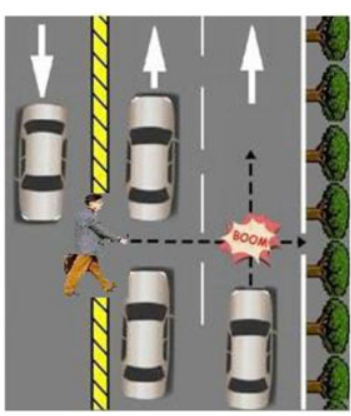

(b)

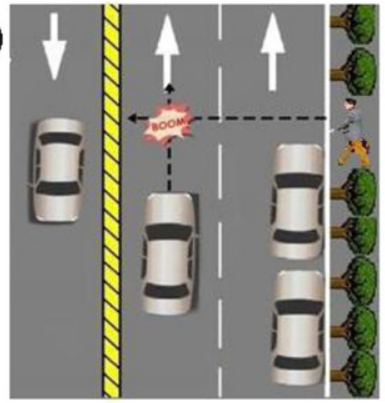

(e)

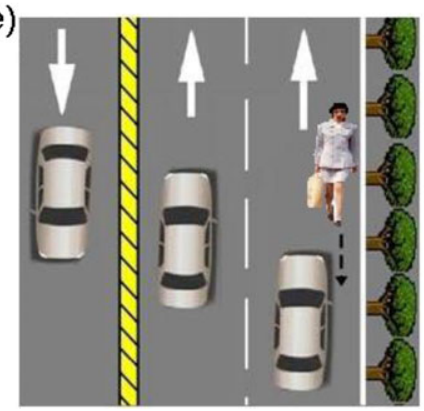

(c)

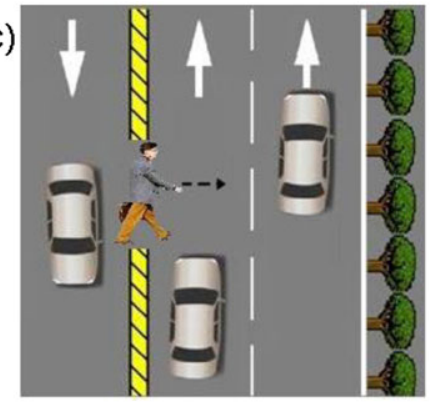

(f)

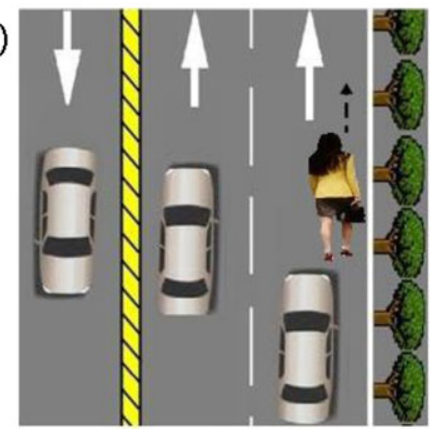

Figure 1. Schematic diagram of the six crash types ((a) nearside crash; (b) nearside dart-out crash; (c) offside crash; (d) offside dart-out crash; (e) facing traffic crash; (f) back to traffic crash).

Data on alcohol use were obtained from the National Health Insurance Database. Blood test results were used with medical record corroboration. Drivers and pedestrians with positive blood alcohol concentrations were identified as driving or walking while drunk. Accurate estimates of the speed at which the vehicles in this study were driven were not available from the data. A vehicle was considered speeding when a police report indicated that the vehicle exceeded the speed limit. Data on the body regions injured in the crashes were obtained from the National Health Insurance Database, which contains imaging data.

\subsection{Analysis}

Crash features and injuries sustained by pedestrians in non-crossing crashes (i.e. facing-traffic and back-to-traffic accident) were compared with those of street-crossing crashes. Increased odds of injury to each body region among injured pedestrians by crash type were estimated using logistic regression controlling for variables that predict the likelihood of the injuries.

\subsection{The binary logit model}

As the outcome responses of interest are binary (i.e. one certain injured region), a common discreteoutcome model, given a set of prediction variables, is the binary logit models. The binary logit model was estimated to evaluate the likelihood of the outcome responses (e.g. 'head injuries' over 'otherwise'; 'chest injuries' over 'otherwise') as a function of crash types, driver/pedestrian behaviours, temporal factors, and roadway characteristics. The theoretical framework of the binary logit model including the model specification and method of evaluation is briefly discussed in this section. Detailed derivation of this model is provided in several studies (e.g. 30).

The binary logit models are widely used if the dependent variable is dichotomous in the regression equation. This model has many advantages over ordinary least-squares regression models, while the dependent variable violates the assumptions of continuous or normal distribution. The logistic regression allows one to predict a binary outcome from a set of explanatory variables that may be continuous, categorical, or a mixture of the two. 


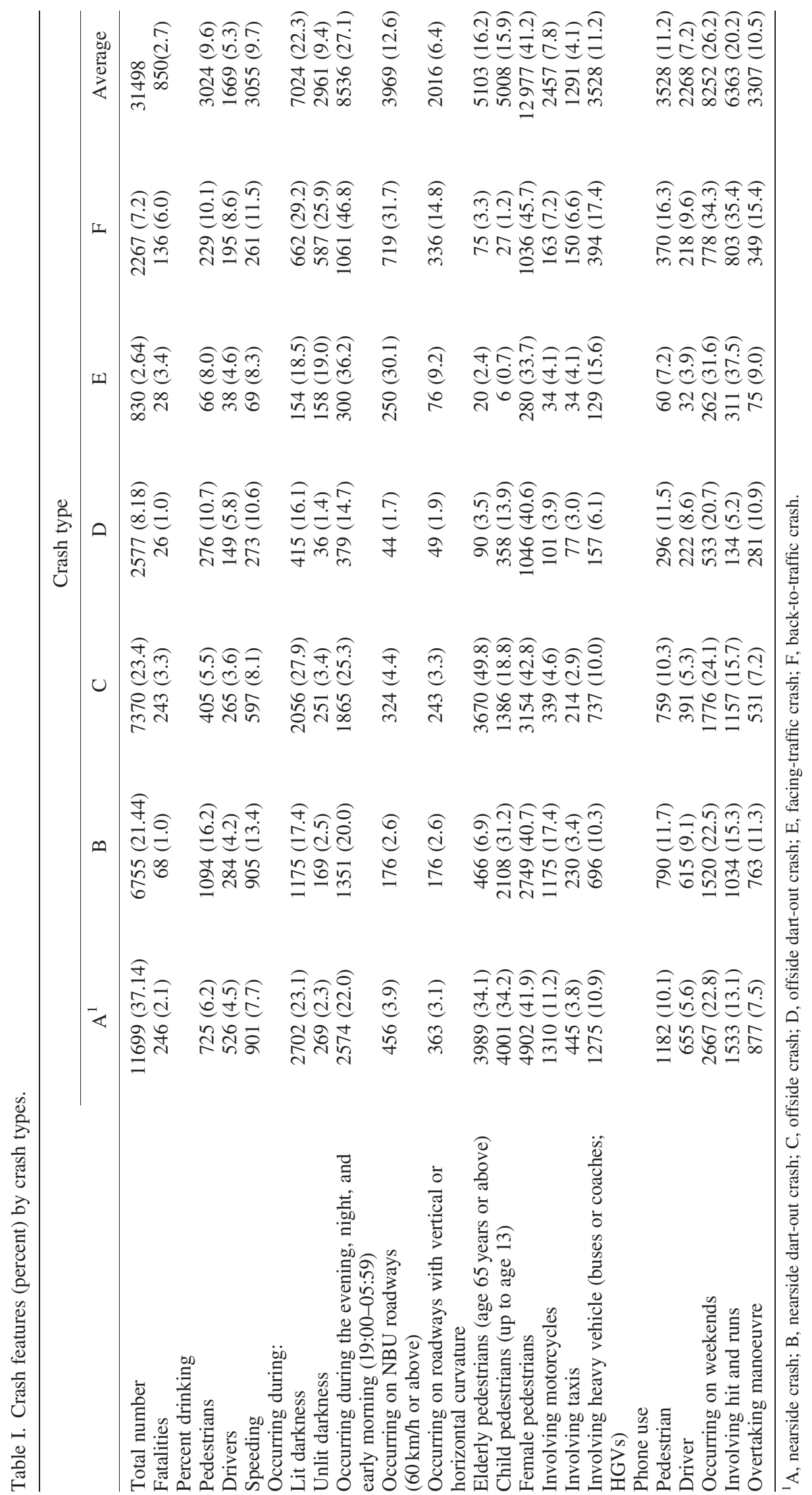


In the logit model, a latent variable is formulated by the following expression:

$$
g(x)=\beta_{0}+\beta_{1} x_{1}+\beta_{2} x_{2}+\ldots+\beta_{j} x_{j}+\ldots \beta_{p} x_{p}
$$

where $x_{j}$ is the value of thejth independent variable and $\beta_{j}$ as the corresponding coefficient, for $j=1,2,3 \ldots p$, and is the number of independent variables.

With this latent variable, the conditional probability of a positive outcome is determined by

$$
\pi(x)=\frac{\exp (g(x))}{1+\exp (g(x))}
$$

The maximum likelihood (ML) method (refer to the work of [30], for a complete discussion of ML estimation in the context of statistical and econometric models) is employed to measure the associations by constructing the likelihood function as follows:

$$
l(\beta)=\prod_{i=1}^{n} \pi\left(x_{i}\right)^{y_{i}}\left(1-\pi\left(x_{i}\right)\right)^{1-y_{i}}
$$

where $y_{i}$ denotes theith observed outcome, with the value of either 0 or 1 , and $i=1,2,3, \ldots, n$, wheren is the number of observations. The best estimate of $\beta$ could be obtained by maximising the log likelihood expression as:

$$
L L(\beta)=\ln (l(\beta))=\sum_{i=1}^{n}\left\{y_{i} \ln \left(\pi\left(x_{i}\right)\right)+\left(1-y_{i}\right) \ln \left(1-\pi\left(x_{i}\right)\right)\right\}
$$

The effect of attribute $k$ on the likelihood of one certain injured region could be revealed by the odds ratio $(\mathrm{OR})$ :

$$
\mathrm{OR}=\exp \left(\beta_{j}\right)
$$

with the $95 \%$ confidence intervals $(\mathrm{CI})$ of $\left(\exp \left(\beta_{j}-1.96 s \beta_{j}\right), \exp \left(\beta_{j}+1.96 s \beta_{j}\right)\right)$, where $s \beta$ is the standard error of the coefficient $\beta$. An OR that is greater than 1 indicates that the concerned attribute leads to a higher probability of one certain injured region, and vice versa. Odds ratios of 1 or close to 1 suggest a neutral or weak effect.

A measure of model goodness-of-fit 'McFadden's pseudo $R^{2}$, [31] can be calculated as

$$
R^{2}=1-\frac{\ln \left(L_{b}\right)}{\ln \left(L_{0}\right)}
$$

where $\ln \left(L_{b}\right)$ is the maximised likelihood and $\ln \left(L_{0}\right)$ is the likelihood assuming all the model slope coefficients are equal to 0 . This measure is bounded by 0 and 1 and as it approaches 1 , model fit improves.

\section{RESULTS}

The crash features in facing-traffic and back-to-traffic accidents were compared with those of other types of street-crossing crashes, as shown in Table I. The average percentage of each variable among the crash configurations is reported in the final column of Table I. Bold numbers represent figures that exceed the average percentage. Accidents involving pedestrians walking along streets represent approximately $10 \%$ of all crashes $(2.64 \%$ for facing-traffic accidents and $7.2 \%$ for back-to-traffic accidents). The most common crash type was nearside accidents, which represent $37.14 \%$ of all 
accidents. Nearside dart-out crashes represent $21.45 \%$ of all accidents, offside crashes represent $23.4 \%$ of all accidents, and offside dart-out crashes represent only $8.18 \%$ of all accidents.

\subsection{Crash features}

Pedestrians in back-to-traffic accidents were fatally injured at a rate of $6.0 \%$, which exceeded the fatality rate associated with other crash types (Table I). The percentage of drunk pedestrians in back-to-traffic accidents was higher than that in facing-traffic accidents (10.1\% vs $8.0 \%)$. Drivers were most often drinking in back-to-traffic accidents (8.6\%), followed by offside dart-out accidents $(5.8 \%)$. Moreover, drivers were most often speeding in offside crashes (13.4\%), followed by back-to-traffic crashes $(11.5 \%)$.

Back-to-traffic accidents causing pedestrian injuries were more likely than any other crash type to occur in darkness (29.2\% of these crashes occurred in lit darkness, and $25.9 \%$ occurred in unlit darkness), and more likely to have occurred during the evening, night, or early morning hours $(46.8 \%$ ), on non-built-up roadways (with speed limits $60 \mathrm{~km} / \mathrm{h}$ or above: $31.7 \%$ ), and on curved roadways (vertical or horizontal: $14.8 \%$ ) than all other crash types.

The percentage of female pedestrians was the highest in back-to-traffic accidents (45.7\%). Up to $37.5 \%$ of pedestrians in facing-traffic accidents were abandoned (i.e. victims of hit-and-run accidents), exceeding the percentage of abandoned pedestrians in all other crash types. Pedestrians in back-totraffic accidents were the second most likely to be abandoned (they were abandoned in $35.4 \%$ of crashes).

Taxies and heavy vehicles were respectively involved in $6.6 \%$ and $17.7 \%$ of back-to-traffic crashes, which exceeded their involvement in other crash types. A total of $16.3 \%$ and $9.6 \%$ of pedestrians and drivers, respectively, in back-to-traffic accidents used phones use, exceeding the phone use among drivers and pedestrians in any other crash type. Furthermore, the percentage of drivers executing overtaking manoeuvres in back-to-traffic accidents was the highest (15.4\%).

Consistent with the findings of past studies (e.g. 4), the current research has provided insights that several crash features were disproportionately represented in facing-traffic and back-to-traffic crashes. For instance, unlit darkness was disproportionately represented in facing-traffic and back-to-traffic accidents $(25.9 \%$ and $19.0 \%$ vs single-digit percentages for the other crossing types of crashes, as shown in Table I). A more specific comparison revealed that the percentages of lit and unlit darkness in back-to-traffic accidents were much higher than those in facing-traffic accidents. These results underscore the importance of street illumination for pedestrian safety. The interaction among unlit darkness and other crash features (e.g. vertical and horizontal street curvature; hit-and-run crashes; early morning, mid night, and evening accidents; and BU/NBU roadways) were further examined in this study (Table II). The average percentage of each variable among the crash configurations is reported in the final row of Table II, and the bold numbers represent figures that exceed the average percentage.

Table II. Crash features (percent) by selected variables, crash types, and BU, and NBU roadways.

\begin{tabular}{|c|c|c|c|c|c|c|c|c|c|c|}
\hline & $\mathrm{BU}$ & & & & & $\mathrm{NBU}$ & & & & \\
\hline Crash & $\begin{array}{l}\text { Vehicle } \\
\text { speeding }\end{array}$ & $\begin{array}{c}\text { Early } \\
\text { morning/ } \\
\text { night/ } \\
\text { evening }\end{array}$ & $\begin{array}{l}\text { Vertical/ } \\
\text { horizontal } \\
\text { curvature }\end{array}$ & $\begin{array}{c}\text { Hit } \\
\text { and run }\end{array}$ & $\begin{array}{c}\text { Unlit } \\
\text { darkness }\end{array}$ & $\begin{array}{l}\text { Vehicle } \\
\text { speeding }\end{array}$ & $\begin{array}{c}\text { Early } \\
\text { morning/ } \\
\text { night/ } \\
\text { evening }\end{array}$ & $\begin{array}{l}\text { Vertical/ } \\
\text { horizontal } \\
\text { curvature }\end{array}$ & $\begin{array}{c}\text { Hit } \\
\text { and run }\end{array}$ & $\begin{array}{c}\text { Unlit } \\
\text { darkness }\end{array}$ \\
\hline $\mathrm{A}^{1}$ & 13.4 & 21.6 & 3.0 & 7.2 & 1.6 & 22.1 & 31.3 & 7.0 & 13.3 & 18.7 \\
\hline B & 9.8 & 19.6 & 2.4 & 20.3 & 2.0 & 16.3 & 24.9 & 9.2 & 25.2 & 23.7 \\
\hline $\mathrm{C}$ & 11.9 & 24.8 & 3.2 & 7.8 & 2.3 & 24.5 & 36.3 & 6.9 & 16.1 & 26.4 \\
\hline D & 9.5 & 14.7 & 1.9 & 3.5 & 1.1 & 19.7 & 13.2 & 4.2 & 5.2 & 17.4 \\
\hline E & 7.0 & 32.3 & 6.5 & 24.6 & 7.7 & 11.6 & 45.3 & 14.6 & 36.9 & 46.7 \\
\hline $\mathrm{F}$ & 9.3 & 40.7 & 14.9 & 31.7 & 9.2 & 13.4 & 59.8 & 21.7 & 40.4 & 61.8 \\
\hline Avg & 10.5 & 25.3 & 4.7 & 15.5 & 4.5 & 18.4 & 35.0 & 10.5 & 22.8 & 33.4 \\
\hline
\end{tabular}

${ }^{1}$ A, nearside crash; B, nearside dart-out crash; C, offside crash; D, offside dart-out crash; E, facing-traffic crash; F, back-to-traffic crash. 
Table II reveals an increase in the percentages of all crash features for NBU roadways compared with those of BU roadways. The percentage of back-to-traffic accidents involving unlit darkness that occurred on BU roadways was $9.2 \%$, whereas $62 \%$ of back-to-traffic accidents that occurred on NBU roadways involved unlit darkness. The percentage of crashes of all types involving unlit darkness on NBU roadways was higher than that for crashes of all types of occurring on BU roadways; this was the most obvious disparity in crash features on NBU and BU roadways. One crucial finding illustrated by Table II is the importance of street lighting for improving pedestrian safety, especially on NBU roadways.

\subsection{Injuries by body region}

The percentages of pedestrians sustaining injuries to different body regions varied among the examined crash types (Table III). Pedestrians in back-to-traffic accidents had the highest percentage of head, neck, and spinal injuries. Moreover, pedestrians in back-to-traffic accidents had the lowest percentage of chest injuries and second lowest percentage of lower extremity injuries.

The odds of head injuries among the pedestrians in facing-traffic or back-to-traffic accidents were 1.36 and 1.86 times those of pedestrians in nearside accidents (Table IV). Compared with those in nearside accidents, the pedestrians in back-to-traffic accidents had more neck and spinal injuries. A decreased prevalence of chest injuries was present when comparing those in back-to-traffic accidents with those in nearside accidents, but no difference was observed in the occurrence of upper and lower extremity injuries between pedestrians in back-to-traffic and nearside accidents. Head injuries, which are generally devastating, were the most frequent injury type among pedestrian victims in facing-traffic and back-to-traffic accidents (Tables IV and V). Specifically, pedestrians in back-to-traffic accidents were more likely than those in facing-traffic accidents to sustain head injuries.

The current research further investigates pedestrian head injuries by evaluating the relative change in the percentage of head injures in various conditions. It is worth investigating whether the percentage of head injuries increases in unsafe conditions such as unlit darkness and NBU roadways (Table VI). The percentage of head injuries was increased among victims injured on NBU roadways relative to those injured on BU to roadways for all crash types, particularly offside dart-out, back-to-traffic, and facing-traffic accidents, for which the percentage of head injuries increased by $179.25 \%$, $150.63 \%$, and $111.72 \%$ respectively. The most apparent disparity between the number of head injuries sustained on NBU roadways and the number of those sustained on BU roadways in unlit darkness conditions was observed in back-to-traffic crashes. For this type of crash, the number of head injuries occurring on NBU roadways exceeded those occurring on BU roadways in unlit darkness conditions by approximately $184 \%$. Pedestrians in back-to-traffic and facing-traffic accidents that occurred during the evening, night, or early morning (19:00-05:00 hours) on NBU roadways respectively experienced the highest and second highest relative changes in the probability of head injuries (approximately $158 \%$ and $125 \%$ respectively).

Table III. Percent of pedestrian body regions injuries by crash type.

\begin{tabular}{lccccccc}
\hline & \multicolumn{7}{c}{ Percent with injuries } \\
\cline { 2 - 7 } Crash type & Head & Neck & Chest & Abdomen & Spine & Upper extremity & Lower extremity \\
\hline $\mathrm{A}^{1}$ & $47.9^{2}$ & 14.1 & 13.7 & 9.1 & 26.2 & 70.4 & 70.6 \\
$\mathrm{~B}$ & 34.4 & 14 & 11.8 & 12.8 & 24.3 & 75.6 & 78.3 \\
$\mathrm{C}$ & 60.6 & 15.4 & 12.3 & 10.9 & 27.2 & 69.4 & 69.8 \\
$\mathrm{D}$ & 33.1 & 13.4 & 11.6 & 9.7 & 22.9 & 66.5 & 73 \\
$\mathrm{E}$ & 62.8 & 11.8 & 8.7 & 9.9 & 24.9 & 68.9 & 70.1 \\
$\mathrm{~F}$ & 77.5 & 16.1 & 5.5 & 10.9 & 35 & 66.9 & 74 \\
Total & 52.7 & 14.1 & 10.6 & 10.6 & 26.7 & 73.2 & 74.5 \\
\hline
\end{tabular}

${ }^{1} \mathrm{~A}$, nearside crash; B, nearside dart-out crash; C, offside crash; D, offside dart-out crash; E, facing-traffic crash; F, back-to-traffic crash.

${ }^{2}$ Pedestrians sustaining injuries to multiple body regions may be counted more than once. 
PEDESTRIANS IN TRAFFIC CRASHES

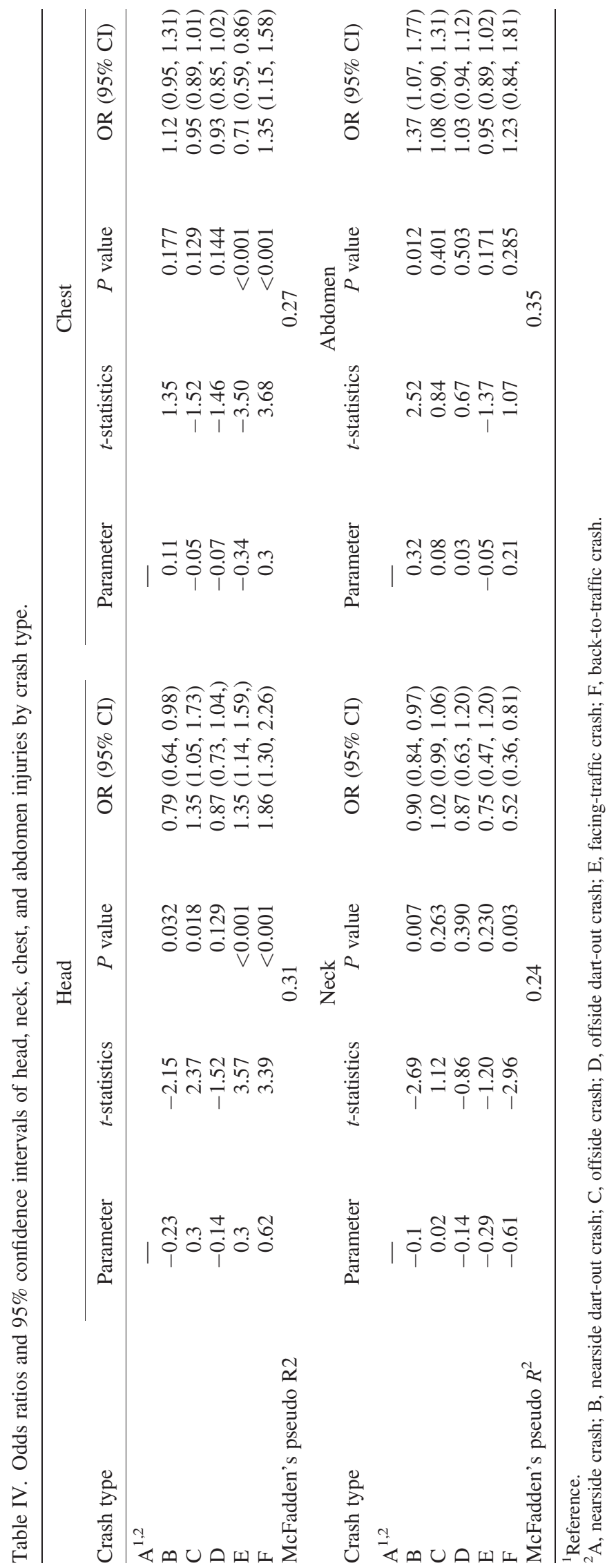




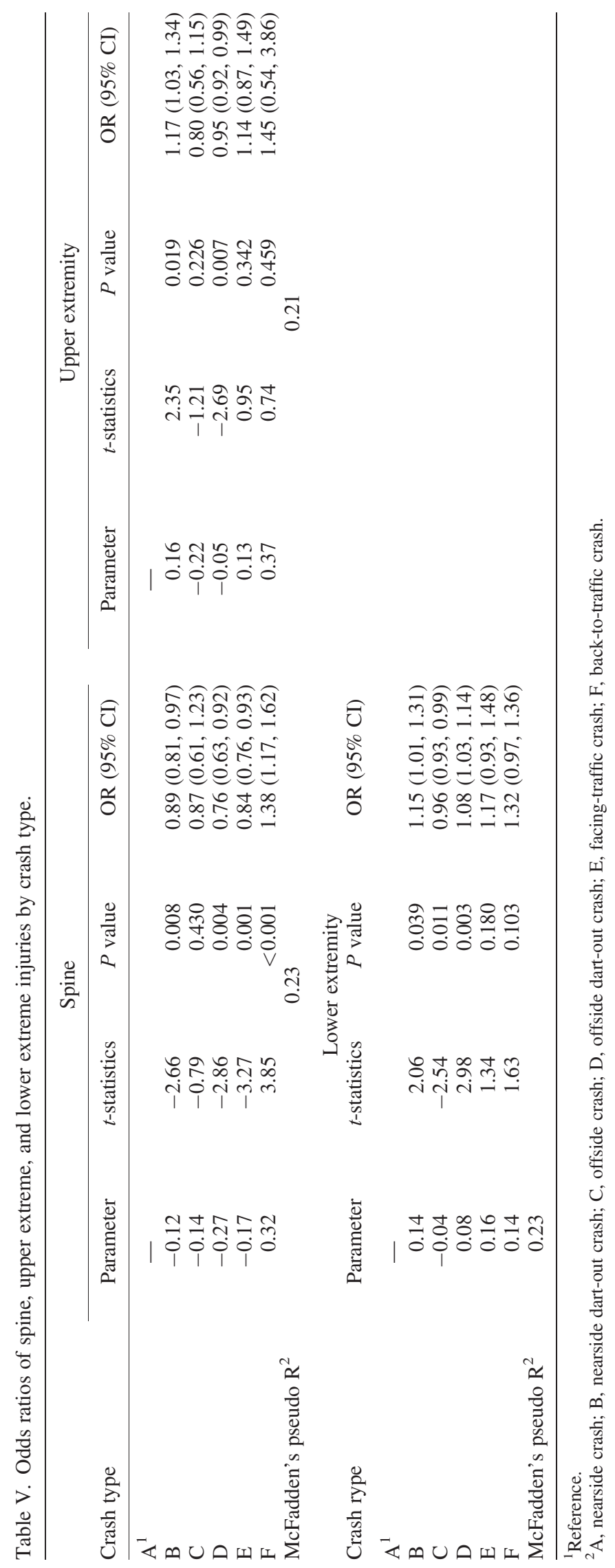


Table VI. Relative change in percentage of head injures in various conditions for accidents occurring on NBU and BU roadways.

\begin{tabular}{lccc}
\hline Crash type & NBU (ref. BU) & $\begin{array}{c}\text { Unlit darkness on } \\
\text { NBU roadways (ref. BU) }\end{array}$ & $\begin{array}{c}\text { Accidents occurring } \\
\text { between 19:00 and 05:59 } \\
\text { on NBU roadways (ref. BU) }\end{array}$ \\
\hline $\mathrm{A}^{1}$ & $32.58 \%$ & $130.06 \%$ & $62.42 \%$ \\
$\mathrm{~B}$ & $46.27 \%$ & $87.77 \%$ & $55.22 \%$ \\
$\mathrm{C}$ & $50.90 \%$ & $85.14 \%$ & $42.06 \%$ \\
$\mathrm{D}$ & $179.25 \%$ & $60.42 \%$ & $65.00 \%$ \\
$\mathrm{E}$ & $111.72 \%$ & $118.02 \%$ & $125.34 \%$ \\
$\mathrm{~F}$ & $150.63 \%$ & $184.13 \%$ & $157.93 \%$ \\
\hline
\end{tabular}

${ }^{1}$ A, nearside crash; B, nearside dart-out crash; C, offside crash; D, offside dart-out crash; E, facing-traffic crash; F, back-to-traffic crash.

The increased odds of head injuries among injured pedestrians by crash type were further estimated using logistic regression controlling for several variables that predict both crash and injury type (Tables VII and VIII). The odds of head injuries among the pedestrians in facing-traffic accidents that occurred on BU roadways were about twice those of pedestrians in nearside accidents. Moreover, the odds of head injuries for pedestrians in back-to-traffic accidents on NBU roadways were 5.69 times those of pedestrians in nearside accidents. Compared with those in nearside accidents, almost all pedestrians in facing-traffic accidents or back-to-traffic accidents (that took place on BU or NBU roadways) had a higher likelihood of sustaining head injuries. The elevated occurrence of head injuries for those in facing-traffic or back-to-traffic accidents was higher for those in accidents that occurred on NBU roadways (i.e. 5.13 and 6.26 respectively for facing-traffic and back-to-traffic accidents occurring in unlit darkness and 2.43 and 2.77 respectively for facing-traffic and back-to-traffic accidents occurring between the hours 19 and 05 ).

\section{DISCUSSION}

The only published study examining the effect of pedestrian walking direction on pedestrian safety reported that facing traffic substantially improves pedestrian safety [4]; pedestrians facing traffic have on average a 77\% lower risk of being struck by a car. The beneficial effect of facing traffic on accident risks (i.e. accident occurrences) was not determined in the current research because of a lack of exposure data. The present study, however, addressed another safety problem: crash features and injuries sustained by pedestrians in various types of accidents. Our data show that pedestrians in back-to-traffic crashes had higher risks of sustaining fatal injuries than did those in facing-traffic crashes. Moreover, back-to-traffic accidents resulted in severe injuries to three anatomical regions, the head, neck, and spine; injuries to these three regions are generally devastating.

The finding that back-to-traffic accidents were more severe than facing-traffic accidents possibly resulted from an increased occurrence of three specific injury types in back-to-traffic accidents: head, neck, and spinal injuries. The most prevalent injuries of these three injuries were head injuries. Back-to-traffic crashes generally result in head injuries (that can generally be severe), and this is particularly prevalent when they occur on NBU roadways in unlit darkness. Likely reasons for the increased occurrence of these head injuries could not be determined in the present study, but an assumption that pedestrians with their backs to traffic are less able to evade vehicles than those who are facing traffic is reasonable.

By conducting multivariate logistics regression and controlling for other variables such as roadway characteristics and time effect, we further investigated head injuries sustained by pedestrians in backto-traffic and facing-traffic crashes in more details (Tables VII and VIII). An elevated risk of head injuries in several circumstances (NBU roadways, unlit streets, and midnight hours) appears evident in our analysis.

The increased risks of head injuries in these two crash types may be attributable to the possibility that drivers not expecting to confront a pedestrian travelling parallel to themselves, in particular in poor-visibility environment in NBU settings. Past studies have established an increased fatality rate 


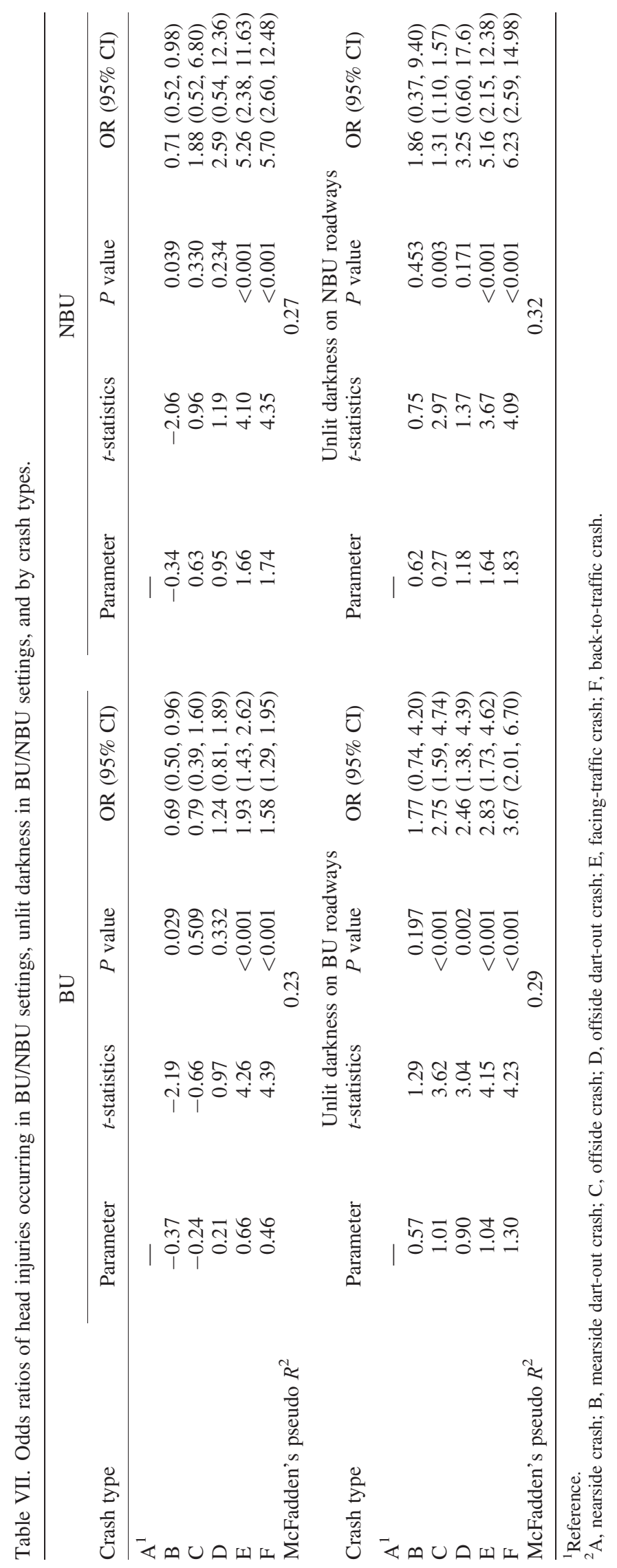




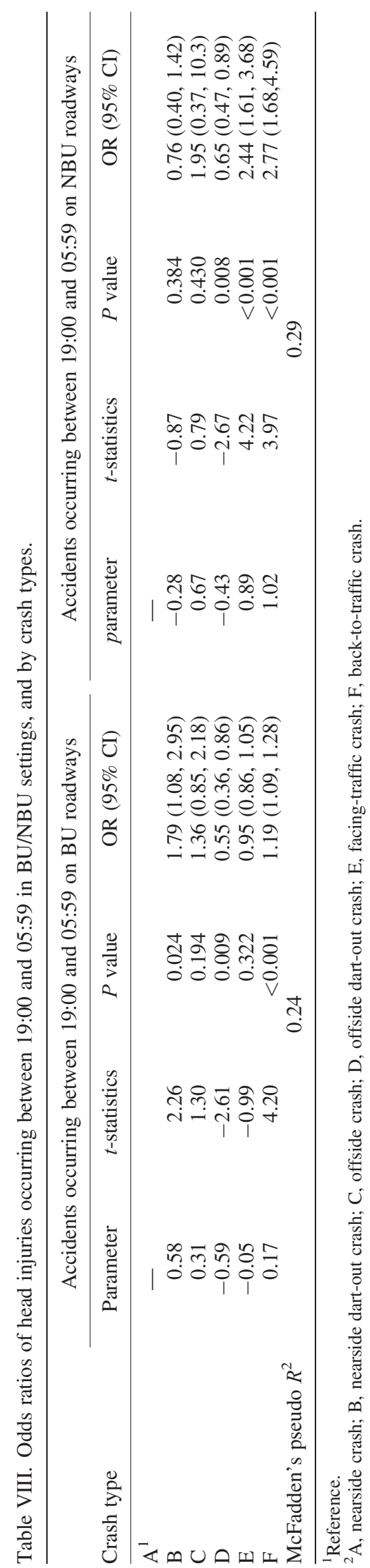


in unlit darkness. In this study, the negative effect of unlit darkness on head injuries was found to be more pronounced on NBU roadways, where drivers may not expect to encounter pedestrians as often as they do on BU roadways. In the current research, unlit darkness seems to act synergistically with other factors (e.g., higher velocities on NBU roadways and walking back to traffic) to increase the risks of head injuries. Indeed, part of our results is consistent with that of Martin et al. [14], who concluded that higher crash impacts may cause the pedestrian to have a higher risk of head injuries that result from multi-impact on the windscreen and ground.

The results also show that when specifically comparing to back-to-traffic crashes, walking facing traffic appears to be more beneficial in decreasing the likelihood of head injuries. This would again point to the benefit of walking facing traffic in terms of accident occurrence and severity (e.g. head injuries). The implication of the findings regarding lit and unlit darkness is that information about the importance of facing traffic should be reinforced to pedestrians who walk in lit or unlit darkness, in particular in NBU settings. In addition, although providing upgrades for roadways such as street lighting and illumination can increase the visibility of pedestrians to drivers (especially on NBU roadways), pedestrians can enhance their visibility to drivers through low-cost measures such as wearing retroreflectors, which have been found to assist drivers in recognising and responding to pedestrians from a safe distance [32, 33]. Increasing street lighting and illumination as well as pedestrians' wearing of retroreflectors may enhance the safety of pedestrians walking along streets, particularly those walking with their backs to traffic.

\section{CONCLUSIONS}

The present study contributes to the literature by addressing the injury patterns in pedestrian back-totraffic and facing-traffic crashes. The main findings include that pedestrians in back-to-traffic crashes had higher risks of sustaining fatal injuries than did those in facing-traffic crashes. Back-to-traffic accidents resulted in a higher risk of severe injuries to three anatomical regions, the head, neck, and spine; injuries to these three regions are generally devastating. Past studies have pointed out the importance of enhancing pedestrian conspicuity in accident occurrence. In the current research, the negative effect of unlit darkness on head injuries (that can normally be devastating) was found to be more pronounced in NBU setting and mid-hour conditions, where drivers may not expect to encounter pedestrians as often as they do on BU roadways and during rush hours. We conclude our study by recommending that in addition to adopting low-cost measures such as wearing retroreflectors at nights, pedestrians should walk facing traffic in any circumstances in particular in the event that sidewalks are unavailable. Facing traffic would benefit in reducing accident risks, as suggested by Luoma and Peltola [4], and our study concludes that facing traffic would reduce the risks of head injuries once an accident has occurred.

It is worthwhile to mention that we extended our univariate injury analysis (refer to the univariate logistic results in Tables IV and V) by estimating the odds of head injury through logistic regression controlling for several variables (Tables VII and VIII). Future work may extend our multivariate analysis by controlling for additional variables (e.g. type of crash partner, geometric characteristics, and human attributes) that were not examined in the present study. In addition, by following our univariate and multivariate analysis procedures, researchers can further investigate the other two frequent injury types (i.e. spinal and neck injuries, which are generally devastating), which are fruitful research topics.

\section{ACKNOWLEDGEMENTS}

The research was funded by the research grants from Ministry of Science and Technology, Taiwan (102-2221-E-038 -017-MY2 and 103-2221-E-038-020-MY2).

\section{REFERENCES}

1. NPA (National Police Administration). Road Traffic and Accident Analysis 2014National Police Administration, Ministry of the Interior: Taipei, Taiwan, 2014. 
2. Fontaine H, Gourlet Y. Fatal pedestrian accident in France: a typological analysis. Accident Analysis and Prevention 1997; 29: 303-312.

3. Rosen E, Sander U. Pedestrian fatality risk as a function of car impact speed. Accident Analysis and Prevention 2009; 41: 536-542.

4. Luoma J, Peltola H. Does facing traffic improve pedestrian safety? Accident Analysis and Prevention 2013; 50: $1207-1210$.

5. Muller B, Rivara F Bergman A. Urban-rural location and the risk of dying in a pedestrian-vehicle collision. Journal of Trauma 1988; 28(1): 91-94.

6. Olszewski P, Szagala P, Wolanski M Zielinska A. Pedestrian fatality risk in accidents at unsignalised zebra crosswalks in Poland. Accident Analysis and Prevention 2015; 84: 83-91.

7. Elvik R, Sorensen M Navestad T. Factors influencing safety in a sample of marked pedestrian crossings selected for safety inspections in the city of Oslo. Accident Analysis and Prevention 2013; 59: 64-70.

8. Dunbar G. Using epidemiological data to address psychological questions about pedestrian behaviour. In Traffic and Transport Psychology: Theory and Application, (Ed)Elsevier: Oxford, 2005 19-25.

9. Schofer J, Christoffel K, Donovan M, Lavigne J, Tanz R Wills K. Child pedestrian injury taxonomy based on visibility and action. Accident Analysis and Prevention 1995; 27: 317-333.

10. Rumar K. The basic driver error: late detection. Ergonomics 1990; 33: 1281-1290.

11. Siddiqui N, Chu X Guttenplan M. Crossing locations, light conditions, and pedestrian injury severity. Transportation Research Record 1982; 2006: 141-149.

12. Sullivan J, Flannagan M. The role of ambient light level in fatal crashes: inferences from daylight saving time transitions. Accident Analysis and Prevention 2002; 34: 487-498.

13. D’Elia A, Newstead S. Pedestrian injury outcome as a function of vehicle market group in Victoria, Australia. Traffic Injury Prevention 2015; 16: 709-714.

14. Martin J, Lardy A Paumon B. Pedestrian injury patterns according to car and casualty characteristics in France. Annals of Advances in Automotive Medicine 2011; 55: 137-146.

15. Mueller B, Nolan J, Zuby D, Rizzo A. Pedestrian injury patterns in the United States and relevance to GTR. IRCOBI Conference 2012. Dublin, Ireland.

16. Hefny A, Eid HO Abu-Zidan F. Pedestrian injuries in the United Arab Emirates. International Journal of Injury Control and Safety Promotion 2015; 22(3): 203-208.

17. Stutts J, Hunter W. Motor vehicle roadway factors in pedestrian and bicyclist injuries: an examination based on emergency department data. Accident Analysis and Prevention 1999; 31(5): 505-514.

18. Paas R, Masson C Davidsson J. Head boundary conditions in pedestrian crashes with passenger cars: sixdegrees-of-freedom post-mortem human subject responses. International Journal of Crashworthiness 2015; 20(6): 547-5593.

19. Maki T, Kajzer J, Mizuno K Sekine Y. Comparative analysis of vehicle-bicyclist and vehicle-pedestrian accidents in Japan. Accident Analysis and Prevention 2003; 35(6): 927-940.

20. Zhao H, Yang G, Zhu F, et al. An investigation on the head injuries of adult pedestrians by passenger cars in China. Traffic Injury Prevention 2013; 14(7): 712-717.

21. Mizuno K, Kajzer J. Head injuries in vehicle-pedestrian impact. SAE Technical Paper 2000, 2000-01-0157.

22. Miles-Doan R. Alcohol use among pedestrians and the odds of surviving an injury: evidence from Florida Law Enforcement Data. Accident Analysis and Prevention 1996; 28(1): 23-31.

23. Ballesteros M, Dischinger P Langenberg P. Pedestrian injuries and vehicle type in Maryland, 1995-1999. Accident Analysis and Prevention 2004; 36(1): 73-81.

24. Henary B, Ivarsson J Crandall J. The influence of age on the morbidity and mortality of pedestrian victims. Traffic Injury Prevention 2006; 7(2): 182-190.

25. Roudsari B, Kaufman R Koepsell T. Turning at intersections and pedestrian injuries. Traffic Injury Prevention 2006; 7(3): 283-289.

26. Sze N, Wong S. Diagnostic analysis of the logistic model for pedestrian injury severity in traffic crashes. Accident Analysis and Prevention 2007; 39(6): 1267-1278.

27. Gabella B, Kathy L, Richard E, Magdalena C Lorann S. Relationship of helmet use and head injuries among motorcycle crash victims in El Paso County, Colorado, 1989-1990. Accident Analysis and Prevention 1995; 27(3): 363-369.

28. Peek-Asa C, Kraus J. Alcohol use, driver, and crash characteristics among injured motorcycle drivers. Journal of Trauma 1996; 41(6): 989-993.

29. Zambon F, Hasselberg A. Factors affecting the severity of injuries among young motorcyclists - a Swedish nationwide cohort study. Traffic Injury Prevention 2006; 7(2): 143-149.

30. Long J. Regression models for categorical and limited dependent variablesSage Publications: Thousand Oaks, CA, 1997.

31. McFadden D. Conditional logit analysis of qualitative choice behaviour. In Frontiers in econometrics, (Ed) Academic Press: New York, 1973.

32. Wood J, Tyrrell R Carberry T. Limitations in drivers' ability to recognise pedestrians at nights. Human Factors 2005; 47: 644-653.

33. Tyrrell R, Wood J, Chaparro A, Carberry T, Chu B Marszalek R. Seeing pedestrians at nights: visual clutter does not mask biological motion. Accident Analysis and Prevention 2009; 41: 506-512. 